\title{
A experiência da Universidade São Francisco com o internato médico de psiquiatria utilizando a metodologia da aprendizagem baseada em problemas
}

\author{
The experience of Universidade São Francisco with the medical internship \\ in psychiatry using the problem-based learning method
}

\author{
Luiz Fernando Ribeiro da Silva Paulin¹, Regina Caeli Guerra Poças ${ }^{2}$
}

${ }^{1}$ Doutor. Professor associado, Disciplina de Psiquiatria, Curso de Medicina, Universidade São Francisco, Bragança Paulista, SP. ${ }^{2}$ Mestranda de Psiquiatria, Universidade Federal de São Paulo (UNIFESP), São Paulo, SP. Professora assistente, Disciplina de Psiquiatria, Curso de Medicina, Universidade São Francisco, Bragança Paulista, SP.

\section{Resumo}

Nos últimos anos, o ensino médico vem sofrendo modificações pedagógicas e curriculares, visando promover a formação de um novo profissional de saúde. Uma das alternativas pedagógicas propostas é a educação problematizadora, que trabalha essencialmente com a construção de conhecimentos a partir da vivência de experiências significativas. Tal modelo de aprendizagem tem viabilizado mudanças curriculares, em que a psiquiatria vem conquistando espaço significativo para o desenvolvimento de uma prática médica mais humanística e integral. O artigo se propõe a discutir a experiência do Curso de Medicina da Universidade São Francisco, em Bragança Paulista (SP) referente à implantação do internato de psiquiatria pela metodologia da aprendizagem baseada em problemas, desde 2003. O internato de psiquiatria ocorre no quinto ano do Curso de Medicina, com carga horária de 320 horas, correspondendo a 8 semanas de 40 horas. As atividades são desenvolvidas no hospital-dia e no ambulatório, e a interconsultoria é realizada no hospital geral. Observa-se significativa evolução no conhecimento adquirido dos alunos, que conseguem, ao término do internato, desenvolver raciocínio clínico e sedimentação de conceitos e práticas psiquiátricas adequadas à formação médica generalista, compatíveis ao que o curso de graduação propõe. A experiência relatada pode servir de modelo para a implantação de uma proposta de aprendizagem em saúde mental inserida nos preceitos da reforma psiquiátrica no Brasil, buscando-se implementar o desenvolvimento de práticas comunitárias e interdisciplinares.

Descritores: Educação médica, método, internato, ensino, psiquiatria.

\begin{abstract}
In the last few years, medical education has been going through curricular and pedagogical changes with the purpose of educating a new health professional. One of the pedagogical alternatives is the education based on problem-solving, which works essentially with the construction of knowledge using significant experiences. Such learning model has enabled curricular changes, which, in the field of psychiatry, reflect the conquest of significant space for the development of a more humane and integral medical practice This article aims to discuss the experience of the Medical School of Universidade São Francisco (USF), in Bragança Paulista, concerning the implementation of the psychiatry internship using the problem-based learning (PBL) methodology since 2003. The psychiatry internship is undertaken in the fifth year of Medical School, and it consists of 320 hours, which correspond to 40 hours a week for 8 weeks. Activities are developed at the day hospital, at the outpatient clinic and at the general hospital during delivery of consultation-liaison psychiatry service. There was significant increase in the knowledge acquired by the students who, upon completing the internship, were able to develop clinical reasoning skills and had consolidated concepts and psychiatric practices adequate to the general medical education and compatible with the purposes of the undergraduate program. The reported experience can serve as a model for the implementation of a learning methodology regarding mental health based on the principles of the psychiatric reformation in Brazil, trying to develop community and interdisciplinary practices.
\end{abstract}

Keywords: Medical education, method, internship, teaching, psychiatry.

Correspondência:

Luiz Fernando Paulin, Rua Francisca Domingues Camargo de Oliveira, 420, Jardim Amapola, CEP 12919-570, Bragança Paulista, SP. Tel.: (11) 4032.0671, Fax: (11) 4034.8398. E-mail: luizpaulin@saofrancisco.edu.br, fernandopaulin@zipmail.com.br

Não há conflitos de interesse associados à publicação deste artigo.

Copyright (C) Revista de Psiquiatria do Rio Grande do Sul - APRS

Recebido em 13/02/2009. Aceito em 17/02/2009. 


\section{Introdução}

Criada em 1971, quando do surgimento de várias escolas médicas no interior do Brasil, a Faculdade de Medicina de Bragança Paulista em nada diferia de outros cursos. Adotava um modelo pedagógico e curricular cujo cerne se baseava no conceito flexneriano, dividindo-se em ciclo básico e clínico, com pouca integração de conhecimentos ${ }^{1,2}$. Com um perfil marcado pela fragmentação, desarticulação e ensino acrítico, observava-se na formação dos alunos o predomínio de atividades teóricas pouco estimulantes durante os 5 primeiros anos do curso. Ficava restrita ao último ano a aprendizagem em serviço, na qual o aluno tinha necessidade de um rápido processo de amadurecimento, além de um treinamento predominantemente hospitalar.

$\mathrm{O}$ internato, no sexto ano, seguia o modelo tradicional em que os alunos passavam pelo sistema de rodízio nas quatro grandes áreas (clínica médica, clínica cirúrgica, ginecologiaobstetrícia e pediatria).

No início dos anos 90, um grupo de professores e alunos, estimulado por um novo projeto socioeducacional implantado pela Universidade São Francisco (USF), iniciou um amplo debate a respeito das diretrizes curriculares do Curso de Medicina e concluiu sobre a necessidade de se elaborar um modelo pedagógico e curricular que viesse ao encontro das proposições fundamentais do projeto. Após quase uma década de discussões e reflexões, o projeto pedagógico começou a ser introduzido no Curso de Medicina da USF, apoiando sua expressão curricular na metodologia da aprendizagem baseada em problemas (ABP).

\section{Algo sobre a ABP}

O modelo tradicional de formação médica há anos tem demonstrado seu esgotamento. Caracteriza-se por um perfil de aluno passivo, preocupado basicamente em conseguir os pontos necessários à sua aprovação, por meio da memorização de informações, quando solicitado ${ }^{3}$. Ao mesmo tempo, cabe ao professor papel centralizador no processo de aprendizagem, tendo nas aulas magistrais o mais importante, senão o único método de ensino. No currículo médico tradicional, há excessivo número de disciplinas, predominantemente teóricas, com pouco tempo para o desenvolvimento de conteúdos e sem critérios de definição quanto à profundidade em ensiná-los. Além disso, as disciplinas são autônomas, ou seja, não ocorre interação entre elas, nem se obedece a um esforço concentrado de formação profissional.

Várias reflexões e ações são realizadas no intuito de buscar uma qualificação ao estudante de medicina, possibilitando-lhe formar-se diante de uma realidade que valorize a equidade, integralidade, interdisciplinaridade e visão crítica, aliadas à competência profissional. Entidades ligadas ao ensino médico, a partir dos anos 80 e principalmente na década de 90, têm procurado descortinar tais mudanças, tornando-se as inovações pedagógicas um dos pilares na formação desse novo profissional de saúde.
Uma das alternativas pedagógicas propostas é a educação problematizadora, que trabalha essencialmente com a construção de conhecimentos a partir da vivência de experiências significativas. Apoia-se nos processos de aprendizagem por descoberta, opondo-se aos de recepção de informações. Os conteúdos de ensino não são oferecidos aos alunos em sua forma acabada, mas no formato de problemas, cujas relações devem ser descobertas e construídas pelo aluno ${ }^{4}$.

Uma das propostas de educação problematizadora é a ABP. Conhecida internacionalmente como PBL, do inglês problem-based learning, a ABP surgiu no cenário educacional, enquanto metodologia de ensino/aprendizagem, no final da década de 60 na McMaster University, em Hamilton, Ontário, Canadá. Após, disseminou-se para outros centros de educação, como as Universidades de Maastricht na Holanda e Harvard e Cornell, nos Estados Unidos. No Brasil, as primeiras escolas que aplicaram tal metodologia foram a Faculdade de Medicina de Marília (1997), a Universidade Estadual de Londrina (1998) e a USF (1999) $)^{5,6}$.

Trata-se de uma estratégia educacional para a aquisição de conhecimentos, habilidades e atitudes, na qual a experiência de ensino ocorre em pequenos grupos. O estudo individual é priorizado e o docente desempenha o papel de facilitador e tutor. O processo de aprendizado (o caminho de conhecimento) é mais valorizado do que o conteúdo (conhecimento) ${ }^{7}$.

Para Cyrino \& Toralles-Pereira ${ }^{4}$, a ABP deve ser considerada como uma das mais significativas inovações na educação médica nos últimos anos. Surgiu como um dos movimentos de reação aos currículos das escolas médicas sob a forte influência do modelo flexneriano que privilegiava o perfil biomédico e o ensino centrado no hospital. "Ela pode ser considerada como o eixo principal do aprendizado teórico de um currículo médico, objetivando o aprendizado de conteúdos cognitivos e a integração de disciplinas. Propõe-se a um trabalho criativo do professor que estará preocupado não só com o 'que', mas essencialmente com o 'porque' e 'como' o estudante aprende"4.

$\mathrm{Na}$ USF, as disciplinas que compõem o novo currículo do Curso de Medicina estão organizadas em: unidade de integração (envolve os conhecimentos essenciais de ciências morfológicas, ciências fisiológicas, medicina interna, clínica cirúrgica, pediatria, gineco-obstetrícia, anatomia patológica, medicina legal e deontologia); unidade de prática médica e sociedade (integra os conhecimentos de epidemiologia, medicina social, medicina do trabalho e bioestatística); unidade de propedêutica médica (engloba os fundamentos da relação médico-paciente, princípios e técnicas de enfermagem e técnicas de semiologia médica); e da disciplina optativa que abrange a complementação dos conhecimentos estimulados nas demais unidades. Essas disciplinas são desenvolvidas nos 8 primeiros semestres do curso. Do primeiro ao quarto semestre, aborda-se a concepção de "homem normal" e do quinto ao oitavo semestre trabalham-se as "patologias e terapêuticas aplicadas".

Os recursos de aprendizagem, além da sala de aula, laboratórios, ambulatórios e hospital, privilegiam a disponibilidade 
de biblioteca que possua um acervo de livros e periódicos, computadores com programas de multimídia, de base de dados, como LILACS e MEDLINE, e conexão com a internet ${ }^{5}$. No entanto, o grande ambiente de aprendizagem ocorre na interface entre o ensino e os serviços de saúde na comunidade, desenvolvendo um novo modelo que desloca o eixo de aprendizado predominantemente hospitalar para o eixo comunitário.

\section{O internato médico da USF no modelo ABP}

Quanto ao internato, o novo projeto pedagógico apresenta a proposta de 2 anos, destacando-se as diretrizes a seguir:

a) Os 2 anos fazem parte de um processo contínuo e articulado em que as competências desenvolvidas se complementam progressivamente de um menor para um maior grau de complexidade.

b) Existem três níveis hierárquicos de atuação (pequena, média e alta complexidade) em que o interno desenvolve seu treinamento através da participação no Programa Saúde da Família, nas unidades básicas de saúde, nos ambulatórios de especialidades e na rede hospitalar.

c) O internato I (quinto ano) possui perfil predominantemente comunitário, enquanto o internato II (sexto ano) realiza atividade centrada em ações hospitalares.

d) O internato I está dividido em seis módulos, a saber:

1 - Saúde do adulto ( 8 semanas);

2 - Saúde da criança (8 semanas);

3 - Saúde da mulher ( 8 semanas);

4 - Saúde mental (8 semanas)*;

5 - Saúde coletiva (6 semanas);

6 - Estágio eletivo (6 semanas).

A carga horária total é de 2.024 horas.

e) No internato II mantém-se o modelo tradicional das quatro grandes áreas. No entanto, foram acrescidos os estágios de urgência - emergência e o estágio eletivo. A carga horária total corresponde a 2.024 horas. A partir de 1999, iniciou-se na USF a primeira turma do Curso de Medicina na metodologia ABP. No ano de 2003, essa turma chegou ao Internato I.

\section{A disciplina de psiquiatria no Curso de Medicina da USF}

\section{O ensino de psiquiatria no modelo tradicional}

Com carga horária pequena (68 horas), a disciplina de psiquiatria do Curso de Medicina da USF era ministrada no quarto ano, e as aulas, exclusivamente teóricas, ocorriam às sextas-feiras à tarde. Como não havia nenhum tipo de atividade prática, no final do curso, então semestral, os alunos eram convidados a visitar um macro-hospital público, em que predominavam pacientes crônicos. Tal situação de ensino, pouco didática, certamente passava ao aluno de graduação a concepção da psiquiatria como uma especialidade basicamente voltada a esse tipo de clientela.

Conforme afirma Dalgalarrondo ${ }^{8}$, citando Shukla et al., a respeito da experiência do ensino de psiquiatria a estudantes de medicina em um hospital especializado clássico: “o hospital psiquiátrico tradicional projetava uma visão distorcida e pessimista da psiquiatria, mostrando aos alunos apenas psicóticos crônicos e deteriorados. Os estudantes voltavam dos hospitais achando a psiquiatria algo interessante mas basicamente inútil e impotente".

\section{O ensino de psiquiatria e o hospital-dia (HD)}

Com o passar dos anos, a disciplina de psiquiatria insistia com os setores competentes do Curso de Medicina e da universidade sobre a necessidade de se criar um serviço, preferencialmente uma enfermaria, no Hospital Universitário. A resposta que se apresentava era a falta de espaço físico para comportar a psiquiatria, bem como outros serviços prioritários.

O discurso oficial refletia não só uma realidade que ocorria na instituição hospitalar, mas também revelava a dificuldade de setores médicos, mesmo ligados à instituição universitária, em aceitarem a existência da psiquiatria em um hospital geral. As resistências internas só foram dirimidas em 1994, com a regulamentação da Portaria FIDEPS ${ }^{\dagger}$, propiciando a instalação de um serviço de psiquiatria no Hospital Universitário São Francisco (HUSF).

Devido à falta de espaço físico, aventou-se a possibilidade de se criar um HD em um espaço anexo à instituição hospitalar, em área de cerca de $300 \mathrm{~m}^{2}$, ao lado de serviços de apoio diagnóstico. Assim foi feito: o HD se instalou ao lado do serviço de quimioterapia e da unidade de pesquisas em farmacologia aplicada à gastroenterologia.

Sem dúvida, o fator desencadeante para a implantação do HD se deu através de estímulos econômicos. Entretanto, não seria esse o momento histórico para viabilizar um projeto ousado em uma instituição de ensino na qual praticamente inexistia a psiquiatria? Não se poderia perder tal oportunidade: adequar os interesses institucionais à criação de um serviço de qualidade, ético e replicador de experiências assistenciais e de formação profissional. Sendo assim, em 25 de janeiro de 1995, foi inaugurado o Hospital-Dia-Escola em Saúde Mental da USF ${ }^{10}$.

Com o surgimento do HD, pôde-se desenvolver mudanças significativas no curso de psiquiatria, embora se mantendo

\footnotetext{
* A partir de 2006, o internato de saúde mental passou para 8 semanas, e o estágio eletivo foi reduzido para 6 semanas.

${ }^{\dagger}$ FIDEPS - Fator de Incentivo ao Desenvolvimento do Ensino e Pesquisa Universitária. Portaria conjunta MS-MEC nº $001 / 94$ foi criada no intuito de que hospitais de ensino que atendessem determinados parâmetros, entre os quais a existência de enfermaria de psiquiatria ou hospital-dia, pudessem obter um adicional de até $75 \%$ sobre o montante pago pelo SUS .
} 
uma pequena carga horária de 68 horas no quarto ano. As aulas teóricas foram substituídas por atividades teórico-práticas. No primeiro momento, os alunos entrevistavam, juntamente com o professor, um paciente e após discutiam o caso embasado no tema a ser desenvolvido naquela aula.

É certo que a existência do HD propiciou alterações ao ensino de psiquiatria. Porém, com uma carga horária pequena, não interagindo com outras disciplinas e desarticulado, replicava o modelo tradicional da formação médica.

\section{O método ABP e o ensino de psiquiatria}

Em 1999, foi implantado o método ABP no Curso de Medicina da USF. A disciplina de psicologia médica, que era ministrada no primeiro ano do curso, foi inserida na Unidade de Propedêutica Médica nos quatro primeiros semestres, com carga horária de 8 horas semanais.

No sétimo semestre, foi instituída a disciplina optativa de propedêutica psiquiátrica, com carga horária de 32 horas. Seu objetivo era apresentar os conceitos básicos da semiologia psiquiátrica, através de entrevistas com os pacientes internados no HD.

Com a perspectiva do internato médico, pôde-se elaborar uma proposta de trabalho em que diretrizes seriam lançadas. Buscava-se a implantação de um programa de internato voltado às propostas apresentadas pelo método $\mathrm{ABP}$, bem como pela experiência acumulada com as atividades de ensino desenvolvidas através do HD.

Vale salientar que trabalhos que discutem a experiência de ensino de psiquiatria no método $\mathrm{ABP}$ são raros $^{11}$, bem como relacionados ao internato ${ }^{12}$.

\section{Internato de psiquiatria}

No ano de 2003, iniciou-se o internato de psiquiatria. Com a carga horária definida em 240 horas/estágio, correspondendo a 6 semanas de 40 horas, significou um aumento de três vezes a carga horária referente à disciplina de psiquiatria no currículo tradicional. A partir de 2006, o internato de psiquiatria passou para 8 semanas, perfazendo o total de 320 horas. Durante o ano, oito grupos com cerca de 10 alunos passam por esse módulo, e alguns alunos optam em repeti-lo enquanto estágio eletivo, tanto no quinto como no sexto ano.

O internato de psiquiatria tem como proposta capacitar a formação do estudante de medicina, com conceitos de saúde mental e dos distúrbios psiquiátricos prevalentes na população brasileira, além de possibilitar o desenvolvimento de práticas interdisciplinares e comunitárias em saúde mental. É certo que o papel desse módulo não é de forma alguma replicar modelos tradicionais disfarçados por metodologias modernas, nem se pautar por um discurso clássico da necessidade de formação de um médico generalista, evidenciando uma prática especialista. $\mathrm{O}$ enfoque principal se converte na exigência de o aluno possuir conceitos em saúde mental que lhe deem condições de atender ao paciente em uma concepção humanística e integral.

$\mathrm{O}$ internato de psiquiatria é centrado em dois eixos básicos de conhecimento e prática, o conceitual e o desinstitucionalizante.

Define-se eixo conceitual como aquele que apresenta conceitos essenciais à elaboração de um raciocínio clínico em saúde mental. Nesse eixo são apresentados temas de psicopatologia, psiquiatria clínica, psicofarmacologia e noções básicas de psicanálise. Tais conhecimentos e sua sedimentação são indispensáveis aos alunos de graduação, mesmo que não venham a ser psiquiatras, visto que os transtornos mentais respondem por $12,3 \%$ das causas de enfermidades e invalidez no mundo. Prevê-se, ainda, uma deficiência de psiquiatras no Brasil, sendo necessário o treinamento de médicos generalistas para o atendimento psiquiátrico básico, devendo-se iniciar, se possível, na graduação ${ }^{13}$.

$\mathrm{O}$ eixo desinstitucionalizante é aquele que traz à tona todo o processo de reestruturação da assistência psiquiátrica no Brasil. Vem sendo pautado pela substituição gradativa do modelo hospitalar psiquiátrico hegemônico por modelos assistenciais em que o $\mathrm{HD}$ e a psiquiatria no hospital geral tornam-se elementos fundamentais. Nesse eixo também é desenvolvida a experiência de práticas interdisciplinares com profissionais não-médicos da equipe e atividades em comum com alunos de outros cursos, como enfermagem e psicologia, que também desenvolvem estágio institucional no HD.

Ainda no eixo desinstitucionalizante, enfatiza-se a necessidade de os alunos treinarem a experiência de práticas que vão além do cenário tradicional (consultório e/ou enfermaria), buscando novos espaços de contato e acompanhamento do paciente. Também é estimulado abranger outros atores que não apenas o paciente, como a família enquanto referência do processo terapêutico.

\section{Funcionalidade}

$\mathrm{O}$ internato está dividido em dois grupos (A e B) que ocorrem em um período de 4 semanas, quando, então, os grupos invertem suas atividades.

O Grupo A desenvolve suas atividades diárias no HD (Tabela 1). Cada aluno é responsável pelo acompanhamento de um a três pacientes, desde o momento de sua internação no serviço. São funções diárias a evolução, a avaliação clínica e a prescrição, sempre sob a supervisão do docente e do residente

As atividades em grupo são o modelo terapêutico predominante em serviços de atenção diária. A participação do aluno permite que ele vivencie formas de atendimento com uma dinâmica própria, por vezes tensionada, em que a relação profissional-paciente apresenta contornos e situações raramente verificadas em atendimentos individualizados. Os alunos

\footnotetext{
‡ O Serviço de Psiquiatria possui Programa de Residência Médica oficial desde 2004. É oferecida uma vaga anual
} 
Tabela 1 - Grupo A (hospital-dia)

\begin{tabular}{|c|c|c|c|c|c|}
\hline Horário & Segunda-feira & Terça-feira & Quarta-feira & Quinta-feira & Sexta-feira \\
\hline 08:00-10:00 & $\begin{array}{l}\text { Grupo de } \\
\text { família }\end{array}$ & $\begin{array}{l}\text { Discussão } \\
\text { de casos }\end{array}$ & Evolução & $\begin{array}{l}\text { Entrevista } \\
\text { com família do } \\
\text { caso clínico }\end{array}$ & $\begin{array}{c}\text { Reunião } \\
\text { clínica }\end{array}$ \\
\hline 10:00-12:00 & $\begin{array}{c}\text { Grupo de } \\
\text { visita médica }\end{array}$ & $\begin{array}{l}\text { Discussão } \\
\text { de casos }\end{array}$ & $\begin{array}{c}\text { Grupo de } \\
\text { visita médica }\end{array}$ & Evolução & $\begin{array}{c}\text { Aula: } \\
\text { psiquiatria clínica }\end{array}$ \\
\hline $13: 00-15: 00$ & $\begin{array}{l}\text { Aula: } \\
\text { psicopatologia }\end{array}$ & $\begin{array}{l}\text { Grupo de } \\
\text { cidadania }\end{array}$ & $\begin{array}{l}\text { Aula: noções } \\
\text { básicas de } \\
\text { psicanálise }\end{array}$ & $\begin{array}{c}\text { Aula: } \\
\text { psicofarmacologia }\end{array}$ & Triagem \\
\hline $15: 00-17: 00$ & $\begin{array}{c}\text { Terapia } \\
\text { ocupacional }\end{array}$ & $\begin{array}{c}\text { Grupo de } \\
\text { visita médica }\end{array}$ & $\begin{array}{c}\text { Aula: } \\
\text { noções básicas } \\
\text { de psicanálise }\end{array}$ & $\begin{array}{c}\text { Aula: } \\
\text { psicofarmacologia }\end{array}$ & Triagem \\
\hline
\end{tabular}

também participam de outras atividades grupais como reunião de família, visita médica, terapia ocupacional e triagem.

Outro momento importante relaciona-se ao grupo de cidadania. Os alunos, sob a supervisão do residente do primeiro ano, desenvolvem temas gerais propostos pelos pacientes, experimentando a possibilidade de coordenar uma atividade grupal. O aluno que está passando no estágio do HD é também o responsável pela preparação do caso clínico a ser discutido em reunião clínica semanal com toda a equipe.
O Grupo B desenvolve suas atividades no ambulatório e no HUSF, onde é realizada a prática de interconsultoria (Tabela 2).

As atividades ambulatoriais ocorrem 2 dias por semana, com casos novos ou retornos em acompanhamento no serviço. Os alunos atendem os casos mais prevalentes de psiquiatria com interesse na formação de um médico geral, tais como transtornos ansiosos, somatizações, depressão e alcoolismo. Casos mais complexos são encaminhados ao ambulatório dos residentes.

Tabela 2 - Grupo B (Ambulatório e Hospital Universitário)

\begin{tabular}{|c|c|c|c|c|c|}
\hline Horário & Segunda-feira & Terça-feira & Quarta-feira & Quinta-feira & Sexta-feira \\
\hline 08:00-10:00 & Interconsulta & $\begin{array}{l}\text { Ambulatório } \\
\text { do município }\end{array}$ & $\begin{array}{l}\text { Ambulatório } \\
\text { do hospital-dia }\end{array}$ & $\begin{array}{l}\text { Ambulatório } \\
\text { do hospital-dia }\end{array}$ & Reunião clínica \\
\hline 10:00-12:00 & Interconsulta & $\begin{array}{l}\text { Ambulatório } \\
\text { do município }\end{array}$ & $\begin{array}{l}\text { Ambulatório } \\
\text { do hospital-dia }\end{array}$ & $\begin{array}{l}\text { Ambulatório } \\
\text { do hospital-dia }\end{array}$ & $\begin{array}{l}\text { Aula: } \\
\text { psiquiatria } \\
\text { clínica }\end{array}$ \\
\hline 13:00-15:00 & $\begin{array}{l}\text { Aula: } \\
\text { psicopatologia }\end{array}$ & $\begin{array}{l}\text { Ambulatório } \\
\text { do município }\end{array}$ & $\begin{array}{c}\text { Aula: } \\
\text { noções básicas } \\
\text { de psicanálise }\end{array}$ & $\begin{array}{c}\text { Aula: } \\
\text { psicofarmacologia }\end{array}$ & Triagem \\
\hline $15: 00-17: 00$ & $\begin{array}{l}\text { Tempo } \\
\text { pró-aluno }\end{array}$ & $\begin{array}{l}\text { Ambulatório } \\
\text { do município }\end{array}$ & $\begin{array}{c}\text { Aula: } \\
\text { noções básicas } \\
\text { de psicanálise }\end{array}$ & $\begin{array}{c}\text { Aula: } \\
\text { psicofarmacologia }\end{array}$ & Triagem \\
\hline
\end{tabular}

$\mathrm{O}$ atendimento do paciente é efetuado pelo interno. Esse, após atendê-lo, discute com o preceptor para posteriormente ambos liberarem o caso. Após, o grupo de internos e o preceptor fazem uma revisão de todos os casos atendidos no dia, destacando-se os pontos mais significativos para a aprendizagem.

Temos evitado seguir em nosso ambulatório a tônica da maioria das escolas médicas, ou seja, a de se criar ambulatórios homogêneos verificando sintomas ou diagnósticos, como, por exemplo, ambulatório de fobias, de transtornos do humor, de esquizofrenia, etc. Nossa opção se dá por se constituir estrutura pequena em que não justificaria nem seria possível uma carga horária ambulatorial tão extensa. Entendemos, ainda, que tais modelos ambulatoriais, apesar de eficientes quanto ao desenvolvimento de pesquisas clínicas, correm o risco de remeter o atendimento a uma prática reducionista. Em outras palavras, escalas de avaliação e critérios diagnósticos se sobreporiam ao atendimento centrado no paciente e não apenas em seus sintomas. As atividades ambulatoriais ocorrem no $\mathrm{HD}$ e no Ambulatório de Saúde Mental de Bragança Paulista, possibilitando a integração de serviços assistenciais do município com a Universidade.

Além do ambulatório, também existe a atividade de interconsultoria no hospital geral. Espécie de "carro-chefe" na integração da psiquiatria com a medicina geral, a interconsultoria tornou-se um importante modelo de inserção da prática psiquiátrica em um espaço em que ela era ignorada, quando não rejeitada ${ }^{14}$. Tem sido viabilizada a internação de pacientes psiquiátricos em leitos de clínica médica, quando possível e indicado.

A interconsultoria ocorre uma vez por semana. $\mathrm{O}$ enfoque que se busca é mostrar a possível integração entre a psiquiatria e outras áreas da medicina.

Como as interconsultas existentes não preenchem o tempo de atividades, opta-se pelo debate de temas prevalentes 
na prática do hospital geral. Delirium, tentativa de suicídio, emergências psiquiátricas, gravidez e puerpério e transtornos alimentares são alguns temas agendados para discussão.

Nesse momento do estágio, os alunos também participam de atividades interdisciplinares, como o atendimento individual familiar, em que se entrevista a família de um paciente internado. A finalidade é aprofundar as noções básicas da dinâmica familiar no contexto dos distúrbios mentais. Tal trabalho é desenvolvido pela assistente social do serviço.

Durante o internato de psiquiatria, preservam-se $15 \%$ da carga horária semanal (6 horas semanais) para o tempo próaluno, um dos pontos fundamentais do método ABP.

Conhecido em algumas escolas médicas pela denominação de "áreas verdes" 13 , o tempo pró-aluno encontra-se inserido desde o primeiro semestre do curso. Tem como objetivo constituir-se em um horário próprio para estudo e solução de situações problemas que se apresentam. No internato de psiquiatria, especificamente, o tempo pró-aluno busca estimular o desenvolvimento de estudo e pesquisas referentes à saúde mental e aos temas propostos para discussão.

\section{Avaliação}

A avaliação é feita sob três formas, a saber:

a) Avaliação conceitual: o corpo docente e a equipe técnica avaliam o aluno pelo seu desempenho e participação nas diversas atividades realizadas. Os critérios são: pontualidade, assiduidade, interesse, iniciativa, responsabilidade, conhecimento teórico e técnico e relação interno-paciente e aluno-docente.

Se no transcorrer do estágio algum interno manifestar dificuldade em desenvolver sua atividade, discute-se o problema verificado no intuito de dirimi-lo.

Essa avaliação tem peso 1 (um).

b) Provas práticas: $\mathrm{o}$ aluno terá duas provas práticas, na quarta e oitava semanas do estágio. Na prova, caso ele esteja passando pelo $\mathrm{HD}$, será discutido o caso que o aluno estiver acompanhando. Se estiver no estágio ambulatorial, será arguido pelo atendimento ambulatorial que fizer no dia da prova.

A banca será composta por pelo menos dois professores da disciplina e o peso de cada prova será 2 (dois).

c) Prova teórica: realizada no final do estágio, essa prova se fundamenta em casos clínicos, estimulando ao aluno o raciocínio clínico psiquiátrico.

Essa avaliação tem peso 5 (cinco).

\section{Conclusão}

Desde sua formação enquanto especialidade médica, a psiquiatria se caracterizou por ser uma especialidade isolada e que se afastou da medicina. Tal situação foi replicada no curso médico da USF, em que ela era oferecida com uma carga horária mínima, em horários inadequados e com atividades predominantemente teóricas. Quando havia atividades práticas, elas ocorriam em macro-hospitais psiquiátricos.

Com a necessidade cada vez mais premente de se pensar e aplicar métodos compatíveis à nova realidade do ensino médico, a educação problematizadora apresenta-se como uma das alternativas metodológicas mais promissoras, pois se encontra fundamentada nos princípios pedagógicos interativo, democrático e pluralista de aprendizado.

O internato de psiquiatria no curso médico da USF, em seu sétimo ano de existência, vem se consolidando a ponto de, a partir de 2006, passar de 6 para 8 semanas. Observase uma significativa evolução no conhecimento adquirido de nossos alunos, que conseguem, ao término do internato, desenvolver raciocínio clínico e sedimentação de conceitos e práticas psiquiátricas adequados à formação médica generalista, compatíveis ao que o curso de graduação propõe. Sinais indiretos de melhora do conhecimento psiquiátrico têm sido observados, tais como encaminhamentos apropriados de pacientes de outras clínicas por alunos que passaram pelo internato de psiquiatria e solicitação de interconsultoria. Observa-se também o número crescente de alunos que desejam fazer estágio eletivo em psiquiatria, bem como maior procura de sextanistas por programas de residência nessa área.

É evidente que tais afirmações devem ser quantificadas. A proposta deste trabalho, no entanto, se resume na apresentação da experiência do internato. Necessita de constante reavaliação tanto por parte dos docentes como dos discentes, no intuito de buscar o aprimoramento na formação médica, garantindo uma prática em que seja superada a segregação ao que sofre de distúrbios mentais.

\section{Referências}

1. Nardy SLM, Polimeno NC, Assis ACB, Martins DC, Gianesella EMF, Lopes LFZ, et al. Projeto pedagógico do curso de medicina da Universidade São Francisco. ABMES. 2000;4:9-28.

2. Taquette SR, Costa-Macedo LM, Alvarenga FBF. Currículo paralelo: Uma realidade na formação dos estudantes de medicina da UERJ. Rev Bras Educ Med. 2003;27(3):171-6.

3. Venturelli J. Educacion medica: nuevos enfoques, metas y metodos. Washington: OPS; 1997.

4. Cyrino EG, Toralles-Pereira ML. Trabalhando com estratégias de ensino-aprendizado por descoberta na área da saúde: a problematização e a aprendizagem baseada em problemas. Cad Saude Publica. 2004;20(3):780-8.

5. Komatsu RS, Zanolli M, Lima VV. Aprendizagem baseada em problemas. In: Marcondes E, Gonçalves E, eds. Educação médica. São Paulo: Servier; 1998. p. 223-37.

6. Projeto pedagógico do curso de medicina. Universidade São Francisco: Bragança Paulista; 2004 (mimeo).

7. Santos SR . O aprendizado baseado em problemas (Problem-Based Learning - PBL). Rev Bras Educ Med. 1994;18(3):121-4.

8. Dalgalarrondo P. Repensando a internação psiquiátrica: a proposta das Unidades de Internação Psiquiátrica no Hospital Geral (dissertação). Campinas: UNICAMP; 1990.

9. Botega NJ. Psiquiatria no hospital geral: histórico e tendências. In: Botega NJ, eds. Prática psiquiátrica no hospital geral: interconsulta e emergência. Porto Alegre: Artmed; 2002. p. 15-30.

10. Paulin LF, Teixeira A, Pereira DQ, Villaça D, Santos DM, Poças RCG. Hospital-dia escola em saúde mental da Universidade São Francisco. Rev Med. USF. 1995;13(1):33-9.

11. McParland M, Noble L, Livingston G. The effectiveness of problem-based learning compared to traditional teaching in undergraduate psychiatry. Med Educ. 2004;38(8):859-67.

12. Hoifodt TS, Olstad R, Sexton H. Developing psychiatric competence during medical education and internship: contributing factors. Adv Health Sci Educ Theory Pract. 2007:12(4):457-73.

13. Coelho BM, Zanetti MV, Lotufo Neto F. Residência em psiquiatria no Brasil: análise crítica. Rev Psiquiatr RS. 2005; 27(1):13-22.

14. Martins LAN, Botega NJ, Celeri EHRV. Interconsulta psiquiátrica. In: Botega NJ, eds. Serviços de saúde mental no hospital geral. Campinas: Papirus ; 1995. p. 55-70. 